\title{
NOVEL CHRONOTHERAPEUTIC MULTIPARTICULATE DRUG DELIVERY SYSTEM OF FELODIPINE: AN EFFECTIVE TREATMENT FOR CARDIAC ARRHYTHMIA
}

\author{
Sabyasachi Banerjee ${ }^{*}$, K. Ravi Shankar ${ }^{2}$, Y. Rajendra Prasad ${ }^{3}$
}

${ }^{1}$ CPS Formulations, Dr.

Reddy's Laboratories Ltd, Hyderabad, India

${ }^{2}$ Sri Sai Aditya Institute of Pharmaceutical Sciences and Research, Surampalem, India 3 College of Pharmaceutical

Sciences, Andhra University, Visakhapatnam, India

Submitted: $12-12-2016$

Revised: $12-01-2017$

Accepted: $15-01-2017$

*Corresponding author

Sabyasachi Banerjee

Email:

sabyasachiph@gmail.com

\begin{abstract}
Arrhythmia follows chronobiology, thus necessitating the development of a time-dependent formulation for its treatment. The aim of the current work was to develop a solubility-enhanced chronotherapeutic system of felodipine, a widely prescribed antiarrhythmic. Systematically optimize hot-melt extrusion process was employed to formulate solubility-enhanced extrudates. The film casting method was adopted for the selection of polymers. Drug released at 5, 15,30min was taken as response variables in $3^{2}$ face-centered cube design. Nearly 10 -fold increase was observed in the solubility of the optimized extrudates in comparison to pure drug. Physical characterization of the extrudates depicted complete amorphization of the drug. The sequential coating was performed onto the extrudates to enable a time-dependent release. In-vitro studies clearly demonstrated that $25 \%$ of the drug was available rapidly within $10 \mathrm{~min}$ of administration. The remaining $75 \%$ of the drug was available over a period of 4,8 and $12 \mathrm{~h}$. Stability studies performed for 6 months at accelerated conditions depicted no significant change in the physicochemical characteristics of the optimized formulation. In-vivo pharmacokinetic studies conducted in beagle dogs ratified the results of in-vitro studies where a sequential time-dependent absorption of felodipine was observed over a period of $12 \mathrm{~h}$. Concisely, the studies demonstrated successful development of a solubility-enhanced chronotherapeutic system of felodipine.
\end{abstract}

Keywords: hot-melt, central composite design, pharmacokinetics, arrhythmia, extrudates

\section{INTRODUCTION}

Cardiac arrhythmia is a serious health problem and has remained a hot topic of scientific discussions since a long time (Evans, et al., 2000; Takahara, et al., 2000; Grant, 2003). Some of the arrhythmias can be mild but if the blood supply to vital organs gets compromised during an arrhythmia, then it can be fatal (Schmid,et al., 2013). Also, unlike many other diseases, the arrhythmia is not a chronic disorder in the true sense. It is well reported in the literature that cardiac arrhythmia follows a distinct chronobiological pattern with attacks likely to occur in late hours of the morning, afternoon and early evening (Peters, et al., 1996). Thus the concentration of drug meant for the purpose should also be at peak in the said time intervals.

One way to achieve this is to administer drug every four hours or so. However, this approach is tedious and it is very difficult to ensure patient compliance with this type of regimens. Due to this, there is a dire unmet need of a drug delivery system capable of delivering the drug at required timings while maintaining the ease of single administration.

Literature reports some of the approaches that have been followed to formulate chronotherapeutic systems of calcium channel blockers (CCBs), one of the most promising therapeutic options for cardiac arrhythmia (Karavas, et al., 2006; Pagar and 
Vavia, 2012). However, these approaches basically focus on combining three to four sequentially coated tablets into one capsule formulation. Moreover, these systems suffer from disadvantages of dose dumping and high inter and intra-subject variability due to their high dependence on gastric emptying. Further, the existing commercial formulation of FLD (Plendil tablets) is based on matrix technology. For matrix type of dosage form, the varying physicochemical characteristics and contractile intensity along the GI tract are the potential sources to alter drug release. Moreover, in the case of matrix type dosage forms the release rate is governed by erosion mechanism which may not be predictable many a times. Due to which, dose dumping and serious adverse events may occur.

Considering these facts, the present study was aimed to formulate multi-particulate chronotherapeutic systems of felodipine. Multiparticulates are less dependent on gastric emptying, resulting in less inter and intrasubject variability in gastrointestinal transit time (Jain, et al., 2008; Roy and Shahiwala, 2009). They are also less prone to local irritation due to their precise distribution of the drug. Also, as the constituent particles pass through GI tract quickly, this result in better pharmacokinetic profiles than single unit dosage forms. Some of the particles, if less than a certain size, can pass even through a closed pylorus. These result in lower intra- and interindividual variability in plasma levels and bioavailability (Abdul, et al., 2010). The present approach focussed on increasing the solubility of felodipine by amalgamating it with Eudragit EPO employing hot-melt approach. The extrudates were differentially coated in order to provide a sequential time-dependent release: around $25 \%$ of the drug to be released immediately and the rest $75 \%$ to be released subsequently at around $4^{\text {th }}, 8^{\text {th }}$, and $12^{\text {th }} \mathrm{hr}$. This would provide a complete therapeutic option for patients with hypertension with the intake of just one capsule. Furthermore, the present work includes a detailed pharmacokinetic study in beagle dogs, a closely related mammal to humans, in order to substantiate our hypothesis. Such a study has not been reported with earlier attempts of chronotherapeutic formulations of felodipine.

\section{MATERIAL AND METHODS}

Felodipine was sourced from Nivedita Chemicals Pvt. Ltd., India. Eudragit EPO was purchased from Evonik Industries, Germany. Soluplus, Povidone, and Copovidone were purchased from BASF, Germany. Ethyl cellulose and dibutylsebacate were obtained from Dow Chemicals, USA and Vertellus Inc., USA. Empty hard gelatin capsule shells were sourced from ACG Capsules, India. All other chemicals employed were of analytical grade.

\section{Evaluation of film casting}

Various polymers (in drug: polymer ratio ranging between 1:1 and 1:5) were screened by film casting method. Briefly, the polymer and the drug substance were dissolved in a common organic solvent and the solvent was vacuum evaporated (Rotary Flash Evaporator, Medica Instrument Mfg. Co., India). The resultant slurry was casted onto a glass surface which was then dried at a temperature of $60^{\circ} \mathrm{C}$. The ensuing film was observed, both physically and microscopically, for surface clarity (i.e., the absence of any drug or polymer particles). The polymers which were evaluated in the study are Copovidone, Soluplus and Eudragit EPO.

\section{Preparation of solid dispersion of felodipine}

Briefly, the blend was prepared by blending $10 \mathrm{mg}$ of drug substance with Eudragit EPO and Povidone K-12 for $10 \mathrm{~min}$ at $25 \mathrm{rpm}$. A co-rotating twin screw extruder (Nano-16, Leistriz, Germany) fitted with a round die, operated at a barrel temperature of $100-130^{\circ} \mathrm{C}$ and screw speed between 100 and $150 \mathrm{rpm}$, was employed to formulate the extrudates of the blend. The resultant extrudates were sized through co-mill to attain a particle size of about $500 \mu \mathrm{m}$. Only those extrudates which were retained above \# 30 ASTM sieve were selected for further process. Table I depicts the detailed composition of the thirteen experimental trials 
formulated as directed by $3^{2}$ face centred cube design (FCCD) (Singh et al., 2013). The formulation at center point was formulated in quintuplicate.

Table I. Levels of Eudragit EPO and Povidone $\mathrm{K}-12$ in the thirteen formulations prepared as per the experimental design;

\begin{tabular}{ccc}
\hline Formulation & $\begin{array}{c}\text { Eudragit } \\
\text { EPO }\end{array}$ & $\begin{array}{c}\text { Povidone } \\
\text { K-12 }\end{array}$ \\
\hline F1 & 10 & 5 \\
F2 & 10 & 5 \\
F3 & 10 & 5 \\
F4 & 30 & 25 \\
F5a & 30 & 25 \\
F5b & 30 & 25 \\
F5c & 30 & 25 \\
F5d & 30 & 25 \\
F5e & 30 & 25 \\
F6 & 30 & 25 \\
F7 & 50 & 45 \\
F8 & 50 & 45 \\
F9 & 50 & 45 \\
\hline
\end{tabular}

Table II. Composition of pulsatile coating solution

\begin{tabular}{lc}
\hline \multicolumn{1}{c}{ Ingredients } & Qty (mg/cap) \\
\hline Ethyl cellulose 22 cps & 7.27 \\
Dibutylsebacate & 0.73 \\
Povidone K-12 & 1.45 \\
Isopropyl alcohol:Water & q.s. \\
\hline
\end{tabular}

\section{In vitro dissolution studies of solid dispersion}

The dissolution of pure drug substance and solid dispersions prepared as per DOE trials were conducted in $900 \mathrm{~mL}$ of $\mathrm{pH} 6.8$ phosphate buffer using USP II apparatus at 50 rpm. Aliquots were collected from the dissolution vessels, filtered through $0.2 \mu \mathrm{m}$ filters and analyzed employing HPLC. The analysis was performed with PDA detector at a $\lambda$ max of $360 \mathrm{~nm}$. X bridge C18, (150 x 4.6mm), the $3.5 \mu \mathrm{m}$ column was employed with a flow rate of $1 \mathrm{~mL} / \mathrm{min}$. An injection volume was $5 \mu \mathrm{L}$ was used. For quantitative determination, a linear calibration graph was obtained over a concentration range of $2.49-99.6 \mu \mathrm{g} / \mathrm{mL}$ with $\mathrm{r}=0.9996$. The Limit of detection and limit of quantification were $0.6 \mu \mathrm{g} / \mathrm{mL}$ and $1.6 \mu \mathrm{g} / \mathrm{mL}$, respectively. Drug released at $5 \mathrm{~min}, 15 \mathrm{~min}$, and 30min (Q5, Q15 and Q30 respectively) were taken as the response variables for the purpose of DoE optimization.

\section{Analysis of DoE data}

Design Expert software (Stat-ease Corporation) was employed to analyze the data obtained from the experimental runs. Response surface graphs and contour plots were generated for better elucidation of the mechanism of action of polymers. An overlay plot was constructed and the formulation which qualified the best to the criteria mentioned below was selected as the optimized formulation. Optimization criteria*: Q5: NLT65\%; Q15: NLT 80\%; Q30: NLT 90\%

*NLT: Not less than

\section{Saturation solubility studies of solid dispersion}

Saturation solubility of felodipine, as well as the optimized solid dispersion, was estimated in buffer solution prepared in the physiological $\mathrm{pH}$ range of 1.2 to 7.0 . Briefly, excess the drug substance or solid dispersion was added into each of the buffers and was subjected to shaking on a mechanical shaker (Remi Laboratory Instruments, India) for $24 \mathrm{~h}$. The samples were collected, filtered through a $0.2 \mu \mathrm{m}$ filter (Millipore, USA) and analyzed employing UV spectroscopy at $365 \mathrm{~nm}$ (UV2600, Shimadzu Corp., Japan).

\section{Characterization of solid dispersion}

\section{XRD studies}

Powder X-ray diffraction (PXRD) (D-8 Advance X-ray diffractometer, Bruker, Germany) studies were conducted for drug substance and the optimized solid dispersion. The study was conducted at a tube voltage and current of $40 \mathrm{kV}$ and $40 \mathrm{~mA}$ respectively. The values of $2 \theta$ ranged between 3 and $45^{\circ} \mathrm{C}$.

Modulated temperature differential scanning calorimetry (MTDSC)

MTDSC analysis of the optimized solid dispersion was performed using differential scanning calorimeter (DSC Q2000, TA 
instruments) for determining the crystals in the solid dispersion. The following parameters were employed: Data storage : off; Equilibrate : At $0.00^{\circ} \mathrm{C}$; Modulate: $+/-1.59^{\circ} \mathrm{C}$ every $60 \mathrm{~s}$ Isothermal : for $5.00 \mathrm{~min}$; Data storage : on; Ramp: $2^{\circ} \mathrm{C} / \mathrm{min}$ to $200^{\circ} \mathrm{C}$; N2 flow : $50.0 \mathrm{~mL} /$ $\min$.

Accurately weighed samples were transferred to the autosampler tray and were subjected to analysis. The thermograms obtained were processed by determining the onset and peak temperature of melting endotherm.

\section{Thermogravimetric analysis (TGA)}

TGA was used to determine the degradation temperature of drug and Eudragit $\mathrm{EPO}$ at a starting temperature of $0^{\circ} \mathrm{C}$, with an increase of $5^{\circ} \mathrm{C}$ up to an end temperature of $250^{\circ} \mathrm{C}$. The nitrogen flow was $60 \mathrm{~mL}$ per min for the sample and $40 \mathrm{~mL}$ per min for the balance.

\section{Coating of solid dispersion Sub-coating}

The optimized solid dispersion extrudates were coated to the level of $5 \%$ with HPMC 6 cps solution in fluidized bed processor (GPCG 1.1, ACG Pam Technologies, India). The coating was performed at a product temperature of $40-45^{\circ} \mathrm{C}$ and atomization of 1.5 bar. The C-type base plate was employed in the coating process. The sub-coated extrudates were divided into two parts: $25 \%$ and $75 \%$. The $75 \%$ fraction was subjected to pulsatile coating.

Pulsatile coating for time-dependent drug release

Seventy-five percent of the sub-coated extrudates (equivalent to $7.5 \mathrm{mg}$ of drug) were further coated with a coating mixture comprising of ethyl cellulose, dibutyl sebacate and Povidone K-12. The quantitative composition is depicted (Table II). Different levels of coating were performed in order to achieve a pulsatile release. One-third of the extrudates were coated to the level of around $6 \% \mathrm{w} / \mathrm{w}$, another one-third of the extrudates were coated to the level of around $10 \% \mathrm{w} / \mathrm{w}$ and the remaining one-third of the extrudates were coated to the level of around $15 \% \mathrm{w} / \mathrm{w}$. All differentially coated fractions, along with the sub-coated extrudates were filled in Size " 3 " hard gelatin capsules.

\section{In vitro dissolution studies of capsules}

The sequential in vitro dissolution studies of the prepared capsule formulation was conducted in USP III apparatus (reciprocating cylinder) at $5 \mathrm{dpm}$ using $250 \mathrm{~mL}$ of dissolution medium. In USP III apparatus, the internal cylinders remain in the first line of vessels containing $0.1 \mathrm{NHCl}$, in the reciprocal movement for $1.5 \mathrm{~h}$. After the programmed period of $1.5 \mathrm{~h}$, the rods rise until the internal cylinders are positioned over the vessels, where they remain for a pre-established timeframe so that the dissolution medium can drain. Then the rods move to the following line containing $\mathrm{pH} 4.5$ acetate buffer, submerging again and the reciprocating actions begin anew for the duration of $30 \mathrm{~min}$. Finally, the rods move to the last line containing $\mathrm{pH} 6.8$ phosphate buffer, submerging into the vessel and reciprocating action continued for $10 \mathrm{~h}$. The samples were analyzed for drug content as per method described under section "In vitro dissolution studies of solid dispersions".

\section{Stability studies}

The optimized formulation was subjected to 6 months of stability studies at accelerated conditions of $40{ }^{\circ} \mathrm{C} / 75 \% \mathrm{RH}$. The formulation was packed in HDPE bottles for the purpose of stability studies. The formulation was analyzed initially and at the end of six months for assay, PXRD, related substances and dissolution profile. Assay and related substance analysis were performed using HPLC with PDA detector at a $\lambda \max$ of $360 \mathrm{~nm}$. $\mathrm{X}$ bridge C18, $(150 \times 4.6 \mathrm{~mm})$, the $3.5 \mu \mathrm{m}$ column was employed with a flow rate of $1 \mathrm{~mL} / \mathrm{min}$. The injection volume was $5 \mu \mathrm{L}$.

\section{In vivo pharmacokinetic studies}

The optimized formulation was subjected to in vivo evaluation in dogs. Taking cognizance that the research work adheres to the guidelines for care and use of the laboratory 
animals, all the animal investigations were performed as per the requisite protocol approved by Dr Reddy's Laboratories. A singledose study was carried out using 12 unisex beagle dogs, with their body weights ranging between 9.21 and $12.70 \mathrm{Kg}$.

The dogs were fasted $12 \mathrm{~h}$ prior to drug administration. All the animals were allowed free access to water throughout the study. Following administration of the optimized encapsulated formulation, dogs were kept in their housing, and access to food and water was allowed ad libitum after 6h. Serial aliquots of blood samples $(1 \mathrm{~mL})$ were withdrawn from each animal at $0.25,0.5,0.75,1,2,4,6,8,10$, $12,14,16,24,48,72,96,120,144,168$, and $192 \mathrm{~h}$ post-dosing and placed in the heparinized tubes. Plasma was harvested by centrifugation (3000rpm, $800 \mathrm{~g}, 5 \mathrm{~min}$ ), and stored at $-20^{\circ} \mathrm{C}$ until analyzed.

The content of felodipine in plasma samples was analyzed by HPLC as per method described under section "In vitro dissolution studies of solid dispersions". Various pharmacokinetic parameters such as plasma concentration-time curve (AUC), maximum drug concentration $\left(\mathrm{C}_{\max }\right)$, the time to reach maximum drug concentration $\left(\mathrm{T}_{\max }\right)$, elimination half-life $\left(T_{1 / 2}\right)$, and elimination rate constant $\left(\mathrm{K}_{\mathrm{e}}\right)$ were calculated employing Win Nonlin software (Version 5.0, M/c Pharsight Corporation, CA, USA).

\section{RESULTS AND DISCUSSION Evaluation of film casting}

The transparency of films prepared with various polymers (i.e. Copovidone, Soluplus and Eudragit EPO) at drug: polymer ratio of 1:5 are compared. Drug-polymer films with Copovidone and Soluplus are opaque whereas clear transparent glassy films are obtained with the Eudragit polymer. Therefore, the Eudragit polymer was selected for further optimization study employing Design of Experiment (DoE) trials.

\section{Analysis of DoE data}

A good fit was observed for the polynomial equation (Eq. 1) used to fit the data with a high value of $\mathrm{R}^{2}$.
$Y=\beta_{o}+\beta_{1} X_{1}+\beta_{2} X_{2}+\beta_{3} X_{1} X_{2}+\beta_{4} X_{1}^{2}+\beta_{5} X_{2}^{2}+\beta_{6} X_{1}$ $\boldsymbol{X}_{2}^{2}+\beta_{7} \boldsymbol{X}_{2} \boldsymbol{X}_{1}^{2} \ldots$ (1)

where $\beta$ o $-\beta 7$ depict the various coefficients and $\mathrm{X} 1$ and $\mathrm{X} 2$ depict the independent variables. Y represents the response variable.

The 3-D response surface graphs and overlay plot for the response variables (Figure 1).

Figure 1 (R1) depicts an inverted U-type curve of Q5 with an increase in the levels of Povidone K-12. The values of Q5 first increase, reach a maximum and then decrease with an increase in the levels of Povidone K-12. The curved lines of the corresponding contour plot also depict that higher levels of Eudragit are imperative for high values of Q5.

As observed in the case of Q5, an inverted U-type curve of Q5 with an increase in the levels of Povidone K-12. Further, in the case of Q15 and Q30, (Figure 1 (R2 and R3)) the similar trend is observed with Eudragit also depicting that optimized levels of both the polymers are imperative for getting adequate dissolution parameters. The corresponding contour plots (not shown in the article) also depict the same.

The optimized formulation is selected by trading-off the values of various responses exhibited the desired values of Q5, Q15 and Q30. The optimized formulation, containing $29.38 \mathrm{mg}$ of Eudragit and $29.06 \mathrm{mg}$ of Povidone K-12, exhibited a Q5 of $69 \%$, Q15 of $85 \%$ and Q30 of 94\%. Figure 1 (overlay plot) depicts the formulation of optimized solid dispersion.

\section{In vitro drug release studies of solid dispersion}

The in vitro drug release of the optimized solid dispersion and pure drug in $\mathrm{pH} 6.8$ phosphate buffer was observed to be $100 \%$ and $5 \%$ respectively, after a duration of $60 \mathrm{~min}$. A nearly 20 -fold increment was observed in the drug release profile of the optimized solid dispersion as compared to the pure drug.

\section{Saturation solubility studies of solid dispersion}

The saturation solubility of optimized solid dispersion and pure drug were observed 

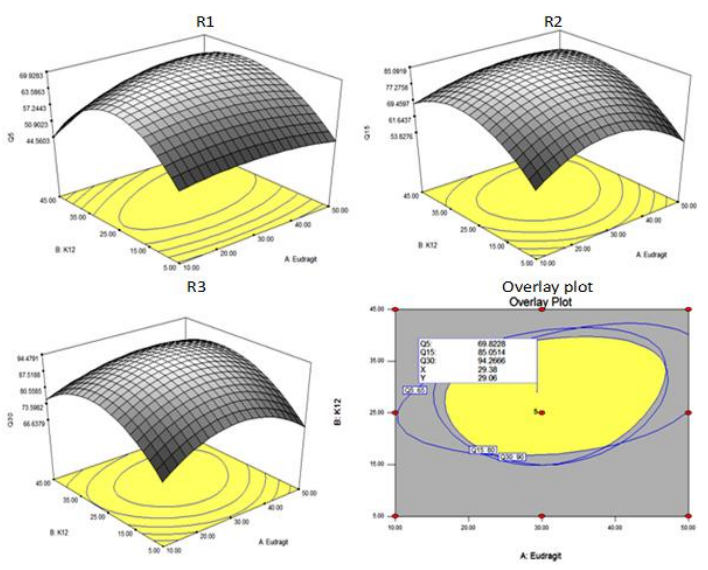

Figure 1. Response surface plot depicting the influence of Eudragit and Povidone on Q5 (R1), Q15 (R2), Q30 (R3) and overlay plot depicting the optimized solid dispersion

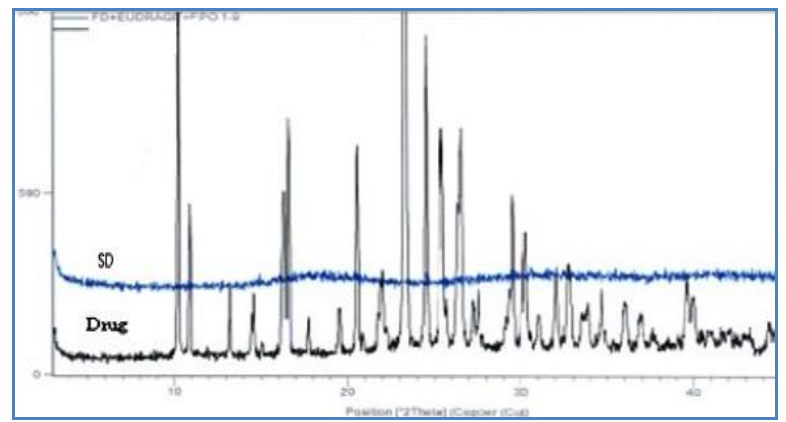

Figure 2. PXRD spectra of pure drug and optimized solid dispersion.

to be $0.047-0.052 \mathrm{mg} / \mathrm{mL}$ and $0.51-0.54$ $\mathrm{mg} / \mathrm{mL}$ respectively. A 10 -fold increase was observed in the solubility of optimized solid dispersion vis-a-vis pure drug in the entire physiological $\mathrm{pH}$ range.

\section{Characterization of solid dispersion}

Figure 2 shows the XRD pattern of pure drug and optimized solid dispersion of FLD and Eudragit in a molar ratio of 1:5. Many high-intensity diffraction peaks were observed in the diffractogram of pure FLD. The numerous distinctive peaks of the pure drug were disappeared in the solid dispersion, indicating conversion of the drug into an amorphous state.

In the DCS thermogram, a single $\mathrm{Tg}$ value ranging from $38.68^{\circ} \mathrm{C}$ to $41.17^{\circ} \mathrm{C}$ is noted for solid dispersion of FLD and Eudragit. This clearly indicates the miscibility of the drug in the polymeric carrier. A decrease in the $\mathrm{Tg}$ value of Eudragit is also observed which notify the plasticizer property of drug in presence of the polymer. TGA study of-of drug and Eudragit indicates that the degradation temperatures of both drug and polymer are around $200^{\circ} \mathrm{C}$, which justifies their suitability for HME process.

\section{In vitro drug release studies of capsule}

The in-vitro drug release profile of final capsule formulation of FLD in sequential dissolution media $(0.1 \mathrm{~N} \mathrm{HCl}$ for $1.5 \mathrm{~h}, \mathrm{pH} 4.5$ buffer for $30 \mathrm{~min}$ and $\mathrm{pH} 6.8$ buffer for $10 \mathrm{~h}$ (Figure 3). Figure 3 clearly showed that around $25 \%$ drug was released at $1 \mathrm{~h}$, around $50 \%$ at $4 \mathrm{~h}$, around $75 \%$ at $8 \mathrm{~h}$ and $100 \%$ at $12 \mathrm{~h}$. 


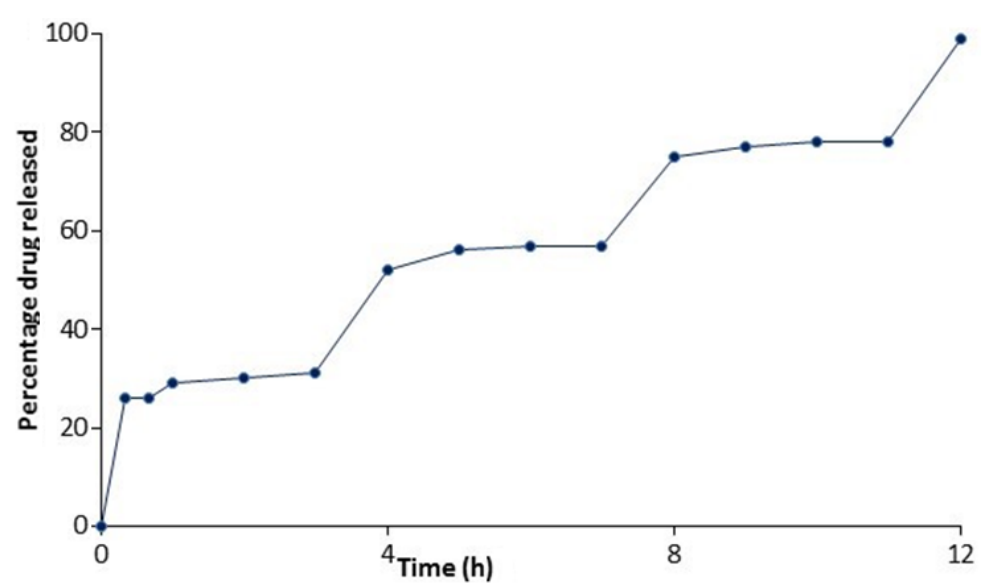

Figure 3.In vitro drug release profile of final capsule formulation

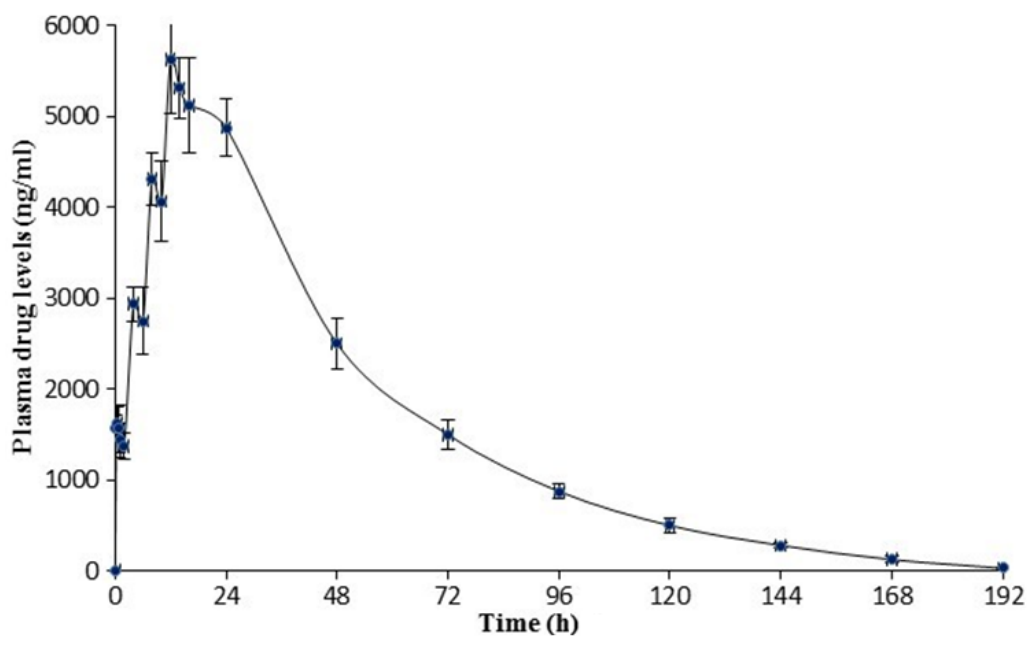

Figure 4. Curve of plasma drug levels as a function of time

Inspection of the reported data reveals that the dissolution rate of the drug is programmed and a constant amount of drug is released initially and an interval of every $4 \mathrm{hr}$ (i.e. $4 \mathrm{~h}, 8 \mathrm{~h}$ and 12h).

\section{Stability studies}

The assay value of the capsule formulation after 6 months of storage at accelerated conditions of $40{ }^{\circ} \mathrm{C} / 75 \% \mathrm{RH}$ was $99.4 \%$ against an initial value of $100.2 \%$. The two identified impurities (Impurity B and C) and all unknown impurities were within the ICH limits at initial and after stability period of 6 months. The PXRD studies of stability samples also showed overlapping spectra with their initial samples. A comparable dissolution profile was observed for the optimized formulation at the initial stage and after 6 months of storage at accelerated conditions.

\section{In vivo pharmacokinetic studies}

The efficiency of the developed formulation was validated by in vivo performance. Table III depicts the values of various pharmacokinetic parameters calculated from the plasma concentration-time profile data. The optimized formulation exhibited a $\mathrm{C}_{\max }$ of $5625.00 \mathrm{ng} / \mathrm{mL}$ and AUC 296.90 ng.hr.mL-1. The $\mathrm{T}_{1 / 2}$ value of $28.39 \mathrm{hr}$ and $\mathrm{K}_{\mathrm{e}}$ 
value of $0.024 \mathrm{hr}^{-1}$ indicate prolonged absorption rate coupled with the slow elimination of the drug from the body. Figure 4 depicts the curve of plasma drug levels as a function of time after $192 \mathrm{~h}$.

Table III. Pharmacokinetic parameter calculated form plasma drug level profile

\begin{tabular}{|c|c|}
\hline Parameter & Value \\
\hline \multicolumn{2}{|c|}{ Primary parameters } \\
\hline $\mathrm{C}_{\max }$ & $5625.00 \mathrm{ng} / \mathrm{mL}$ \\
\hline $\mathrm{AUC}_{0-\mathrm{t}}$ & 296.90ng.h.mL-1 \\
\hline \multicolumn{2}{|c|}{ Secondary parameters } \\
\hline $\mathrm{T}_{\max }$ & $12.21 \mathrm{~h}$ \\
\hline$K_{e}$ & $0.02 \mathrm{~h}^{-1}$ \\
\hline $\mathrm{T}_{1 / 2}$ & $28.39 \mathrm{~h}$ \\
\hline
\end{tabular}

Like a majority of drug substances, Felodipine, a promising CCB, suffers from a major downside of poor solubility (Raina et al., 2014; Sarode et al., 2014a; Sarode et al., 2014b). Since the drug belongs to Class II as per the Biopharmaceutics Classification system (Tapas et al., 2009), permeability is not the rate limiting step in its absorption. Thus, apart from the first-pass metabolism, one more predicament governing the low bioavailability of felodipine is its very low solubility in the lumen. Considering this fact, reports abound in the literature describing various attempts to increase the solubility and dissolution of the drug substance (Karavas et al., 2005; Alonzo et al., 2011; Basalious et al., 2011). Approaches like nanodispersion (Karavas et al., 2006) and $\beta$ cyclodextrin(Pagar and Vavia, 2012) complexation have been employed for the purpose. However, neither of the two approaches are effective enough to render the drug substance completely amorphous. Complete amorphization of drug essential for ensuring complete absorption, particularly, in the case of felodipine since it suffers from very low solubility. The increased solubility also helps in reducing intra and inter-individual variabilities in drug absorption as the only solubility is the rate-limiting step in its absorption.
The film-forming capacity of various polymers depends upon their ability to form a transparent glassy solution with the drug. Copovidone and Soluplus were not able to form a clear glassy film as the quantity of the polymer employed was not sufficient to induce molecular interactions. On the other hand, Eudragit was able to form a clear transparent glassy film in the same drug: polymer ratio (1:5). The same can be attributed to the flexible chains present in the polymer which accommodate themselves to form a clear matrix at a temperature as low as $40-45^{\circ} \mathrm{C}$. This property, in particular, has been imparted by the low glass transition temperature ( $\mathrm{Tg}$ of around $45^{\circ} \mathrm{C}$ ) of Eudragit EPO (Higashi et al., 2014). As only Eudragit EPO was able to form a transparent film, the same was selected for further optimization employing DoE. Also, the extrudates had to withstand the rigorous fluidization during the coating process. Since, many-a-times, a binder is also required in order to provide adequate strength to the extrudates, it was decided to introduce a low-viscosity binder, Povidone $\mathrm{K}-12$, in the optimization trials. The effect of this additional binder on the dissolution characteristics of extruding was envisaged to be studied employing a detailed FCCD.

A $3^{2} \mathrm{FCCD}$ was employed in the current development work because it provides the benefit of exploring quadratic interactions between the polymers (Singh et al., 2009; Dhawan et al., 2011; Singh et al., 2011). Further, the formulation at center point was formulated in quintuplicate and analyzed five times to ensure the robustness of the design. Such is not feasible with standard factorial designs (Kapil etal., 2013). The high values of correlation coefficient vouched for adequate selection and the high prognostic ability of the design.

The increase in the rate of dissolution of the drug with high levels of Eudragit can be attributed to the ability of the polymer to disperse the drug into amorphous particles or molecular dispersion (Feng et al., 2012). Further, Eudrgait EPO prevents the recrystallization by stabilizing the drug substance through hydrogen bonding, vander Waal and ionic interactions 
(Higashi et al., 2014). Povidone, on the other hand, provided a synergistic effect in drug release.

A nearly 20-fold increment was observed in the drug release profile of the optimized solid dispersion as compared to the pure drug. This proves the adequate selection of the solid dispersing polymer, the experimental design, and our hypothesis. The same is also corroborated by saturation solubility studies where a drastic increase in the solubility of drug substance is observed upon being formulated as a solid dispersion.

The absence of any crystalline peak in the PXRD spectra clearly corroborates the complete conversion of crystalline felodipine to its amorphous form (Rezaei Mokarram et al., 2010; Pattnaik et al., 2011). This is because Eudragit EPO was able to disperse the drug substance on a molecular level leading to an amorphous product that exhibits a single glass transition temperature.

MTDSC is an important tool to assess the amorphous content of the sample (Gill $e t$ al., 2010). Since the sensitivity of MTDSC is more as compared to conventional DSC, it can even estimate the amount of molecularly dispersed drug. A single $\mathrm{Tg}$ in DSC thermogram clearly indicates that no phase separation has taken place and the drug is molecularly dispersed in the polymer. TGA studies showed high degradation temperatures of both drug and polymer, signaling that the temperature at which the hot melt process is carried out is not at all detrimental to the stability of the product.

The in vitro dissolution profile clearly demonstrates that a time-dependent sequential drug release is observed when the optimized capsule formulation was subjected to dissolution studies. Thus the concentrations of which the different extrudates were coated were successfully able to control the release of drug in the programmed hours. A nearly equal amount of drug was released initially, and at $4^{\text {th }}$, $8^{\text {th }}$ and $12^{\text {th }} \mathrm{hr}$. The coating polymer, ethyl cellulose does not have a solubility in aqueous media, thus providing complete protection till the layer is intact. Dibutyl sebacate, in a generally acceptable concentration of $10 \% \mathrm{w} / \mathrm{w}$ (Alavi et al., 2002), was able to provide enough plasticity to the film in addition to providing a synergistic release-controlling effect because of its hydrophobicity. As the thickness of coat increased, the drug release was observed to be decreased proportionally. This can be attributed to the ethyl cellulose layer becoming more impermeable to dissolution media. Povidone $\mathrm{K}-12$ acted as a pore-former in the formulation thus helping in the release of drug (Zhang et al., 2007; Tuntikulwattana et al., 2010; Yang et al., 2014). On coming in contact with the dissolution medium, Povidone gets dissolved thus leaving behind a pore in the coat. This facilitates the drug release without affecting the flexibility of the film.

Stability studies concluded that there was no significant change observed in the assay, dissolution profile, impurities level and polymorphic form of the optimized formulation upon storage at accelerated condition of $\quad 40{ }^{\circ} \mathrm{C} / 75 \% \mathrm{RH}$ for a period of 6 months. This further emphasizes the robustness and ruggedness of developed formulation.

The in vivo pharmacokinetic studies are in agreement with the anticipated results. There was a strong correlation between the results obtained during in vitro dissolution studies and the in vivo pharmacokinetic studies. As can be discernible from Figure 4, a time-dependent sequential absorption of drug was observed over a period of $12 \mathrm{~h}$. Since permeability of felodipine was never a point of concern, the two factors crucial in developing a successful chronotherapeutic systems of felodipine were its rate of drug release and the solubility of released drug in intestinal lumen. One of the factors was successfully addressed by adequately optimized hot melt extrusion process while the other was taken care by a precisely designed sequential coating system. Since this is a long half-life drug, the subsequent dose of drug was absorbed much before majority of drug being eliminated. This gives rise to the characteristics peaks and troughs in the plasma drug level profiles, thus maintaining the drug levels at therapeutic 
concentrations throughout the therapy. The value of half-life (around $28 \mathrm{hr}$ ) is in close agreement with previously reported literature. Due to this long half-life of the drug, it took $192 \mathrm{hr}$ to achieve near complete elimination. The in vitro drug release is successfully translated in the in vivo conditions, thus establishing a proof of concept formulation for sequential release of felodipine.

\section{CONCLUSION}

In a nutshell, the present study demonstrates the successful development of a solubility-enhanced chronotherapeutic system of felodipine capable of providing a timedependent drug availability in blood. The said system can be used as single therapy for daylong management of arrhythmia.

\section{ACKNOWLEDGEMENT}

The authors gratefully acknowledge the CPS-Formulations department of Dr. Reddy's Laboratories Ltd., Hyderabad, India for providing the requisite research facilities. Vital inputs from Rishi Kapil are gratefully acknowledged.

\section{REFERENCES}

Abdul S., Chandewar AV. Jaiswal SB., 2010. A flexible technology for modified-release drugs: multiple-unit pellet system (MUPS). J Control Release. 147: 2-16.

Alavi AK., Squillante E., Mehta KA., 2002. Formulation of enterosoluble microparticles for an acid labile protein. $J$ Pharm Pharm Sci. 5: 234-44.

Alonzo DE., Gao Y., Zhou D., Mo H., Zhang, GG., Taylor LS., 2011. Dissolution and precipitation behavior of amorphous solid dispersions. J Pharm Sci. 100: 331631.

Basalious EB., El-Sebaie W., El-Gazayerly O., 2011. Application of pharmaceutical QbD for enhancement of the solubility and dissolution of a class II BCS drug using polymeric surfactants and crystallization inhibitors: development of controlled-release tablets. AAPS PharmSciTech. 12: 799-810.
Dhawan S., Kapil R., Singh B., 2011. Formulation development and systematic optimization of solid lipid nanoparticles of quercetin for improved brain delivery. J Pharm Pharmacol. 63: 342-51.

Evans SJ., Dalton GR., Levi AJ., 2000. Experimental studies on myocardial stretch and ventricular arrhythmia in hypertrophied and non-hypertrophied hearts. J Cardiovasc Risk. 7: 163-75.

Feng J., Xu L., Gao R., Luo Y., Tang X., 2012. Evaluation of polymer carriers with regard to the bioavailability enhancement of bifendate solid disper-sions prepared by hot melt extrusion. Drug Dev Ind Pharm. 38: 735-43.

Gill P., Moghadam TT., Ranjbar B., 2010. Differential scanning calorimetry techniques: applications in biology and nanoscience. J Biomol Tech. 21: 167-93.

Grant AO. 2003. Recent advances in the treatment of arrhythmias. Circ J. 67: 651-5.

Higashi K., Yamamoto K., Pandey MK., Mroue KH., Moribe K., Ramamoorthy A., 2014. Insights into the atomic-level interaction between mefenamic acid and eudragit EPO in a supersaturated solution by highresolution magic-angle spinning NMR spectroscopy.Mol Pharm. 11: 351-7.

Jain SK., Agrawal GP., Jain NK., 2008. Floating microspheres as drug delivery system: newer approaches. Curr Drug Deliv. 5: 220-3.

Kapil R., Dhawan S., Beg S., Singh B., 2013. Buccoadhesive films for once-a-day administration of rivastigmine: systematic formulation development and pharmacokinetic evaluation. Drug Dev Ind Pharm. 39: 466-80.

Karavas E., Georgarakis E., Bikiaris D., 2006. Felodipine nanodispersions as active core for predictable pulsatile chronotherapeutics using PVP/HPMC blends as coating layer. Int J Pharm. 313: 189-97.

Karavas E., Ktistis G., Xenakis A., Georgarakis, E., 2005. Miscibility behavior and formation mechanism of stabilized felodipine-polyvinylpyrrolidone 
amorphous solid dispersions. Drug Dev Ind Pharm. 31: 473-89.

Pagar KP., Vavia PR., 2012. Felodipine betacyclodextrin complex as an active core for time delayed chronotherapeutic treatment of hypertension. Acta Pharm. 62: 395-410.

Pattnaik S., Swain K., Mallick S., Lin Z., 2011. Effect of casting solvent on crystallinity of ondansetron in transdermal films. Int J Pharm. 406: 106-10.

Peters RW., Brooks MM., Zoble RG., Liebson, PR., Seals AA., 1996. Chronobiology of acute myocardial infarction: cardiac arrhythmia suppression trial (CAST) experience. Am J Cardiol. 78: 1198-201.

Raina SA., Zhang GG., Alonzo DE., Wu J., Zhu D., et al., 2014. Enhancements and limits in drug membrane transport using supersaturated solutions of poorly water soluble drugs. J Pharm Sci. 103: 2736-48.

Rezaei MA., Kebriaee ZA., Keshavarz M., Ahmadi A., Mohtat B., 2010. Preparation and in-vitro evaluation of indomethacin nanoparticles. Daru. 18: 185-92.

Roy P. Shahiwala A., 2009. Multiparticulate formulation approach to pulsatile drug delivery: current perspectives. I Control Release. 134: 74-80.

Sarode AL., Malekar SA., Cote C., Worthen DR., 2014a. Hydroxypropyl cellulose stabilizes amorphous solid dispersions of the poorly water soluble drug felodipine. Carbohydr Polym. 112: 512-9.

Sarode AL., Wang P., Obara S., Worthen DR., 2014b. Supersaturation, nucleation, and crystal growth during single- and biphasic dissolution of amorphous solid dispersions: polymer effects and implications for oral bioavailability enhancement of poorly water soluble drugs. Eur J Pharm Biopharm. 86: 351-60.

Schmid MB., Hopfner RJ., Lenhof S., Hummler, HD., Fuchs H., 2013. Cerebral desaturations in preterm infants: a crossover trial on influence of oxygen saturation target range. Arch Dis Child Fetal Neonatal Ed. 98: F392-8.
Singh B., Kapil R., Nandi M., Ahuja N., 2011. Developing oral drug delivery systems using formulation by design: vital precepts, retrospect and prospects. Expert Opin Drug Deliv. 8: 1341-60.

Singh B., Pahuja S., Kapil R., Ahuja N., 2009. Formulation development of oral controlled release tablets of hydralazine: optimization of drug release and bioadhesive characteristics. Acta Pharm. 59: 1-13.

Singh B., Singh R., Bandyopadhyay S., Kapil R. Garg B., 2013. Optimized nanoemulsifying systems with enhanced bioavailability of carvedilol. Colloids Surf $B$ Biointerfaces. 101: 465-74.

Takahara A., Sugiyama A., Dohmoto H., Yoshimoto R., Hashimoto K., 2000. Antiarrhythmic and cardiohemodynamic effects of a novel $\mathrm{Ca}(2+)$ channel blocker, AH-1058, assessed in canine arrhythmia models. Eur J Pharmacol. 398: 107-12.

Tapas AR., Kawtikwar PS., Sakarkar DM., 2009. Enhanced dissolution rate of felodipine using spherical agglomeration with Inutec SP1 by quasi emulsion solvent diffusion method. Res Pharm Sci. 4: 77-84.

Tuntikulwattana S., Mitrevej A., Kerdcharoen T., Williams DB., Sinchaipanid N., 2010. Development and optimization of micro/nanoporous osmotic pump tablets. AAPS PharmSciTech. 11: 924-35.

Yang M., Xie S., Li Q., Wang, Y., et al., 2014. Effects of polyvinylpyrrolidone both as a binder and pore-former on the release of sparingly water-soluble topiramate from ethylcellulose coated pellets. Int J Pharm. 465: 187-96.

Zhang X., Wang Y., Wang J., Li, S., 2007. Effect of pore former on the properties of casted film prepared from blends of Eudragit NE 30 D and Eudragit L 30 D-55. Chem Pharm Bull (Tokyo). 55: 12613 . 